\title{
Intelligent Traffic Control System For Emergency Vehicle
}

\author{
Minal Patil $^{\# 1}$, Abhishek Madankar ${ }^{\# 2}$, Vivek Pise ${ }^{\# 3}$ \\ ${ }^{1,2}$ Department of Electronics and Telecommunication Engineering, RTMNU, India \\ ${ }^{3}$ Department of Electronics Engineering, GONDWANA University, India \\ 15.mpatil@gmail.com \\ 2vicky.madankar123@gmail.com \\ ${ }^{3}$ vivek_pise@rediffmail.com
}

\begin{abstract}
The proposed design is based on an traffic controlling, which allow high priorities vehicles like ambulance. Each ambulance is configured with zigbee module (placed at a predefined location). In this module PICAT89C52 system-on-chip module uses to read the ZIGBEE module attached to the vehicle. It recognizes the time span of green light of respective path. As the ambulance is heading toward the junction, the green signal will turn ON. The signal will turn ON by communication with the traffic controller which is placed at junction. With the help of Zigbee modules and PICAT89C2051, System on chip wireless communication takes place between ambulance and traffic controller. The module is tested and the experimental results were found is expected.
\end{abstract}

Keyword- PICAT89C205, PIC89C52, MAX232, ULN2803A, ZigBee

\section{INTRODUCTION}

INDIA is one of the fast growing economies in the world and it is second most populous Country. In india traffic congestion problem happens frequently. Day by day number of vehicles are increasing exponentially but to cope up with this exponential growth india does not have any traffic control mechanism. Also, the traffic in indian is disordered and it is non lane based. For solving such problems it required very accurate traffic control system. ZigBee Technology can be used in intelligent traffic junction. Generally the ZigBee module is very capable to transmit data over long distance with the help of Mesh topology. As per the specification of ZigBee module, it is having defined rate of $250 \mathrm{Kbit} / \mathrm{s}$, that rate is very suitable for transmission of data through sensor or input devices.

\section{LITERATURE SURVEY}

In Green wave technology the path of emergency vehicle will be cleared by just turning all the red light of that path into green light. In this technology the main drawback of this system is synchronization. If the synchronization not takes place properly, the mob of vehicles increases and it will form a long queue due to which green wave grow in size until it becomes too large due to which some vehicles get struck in the traffic and they cannot reach in time. This is called over-saturation road junction areas. This system will definitely provides very effective time management system. It will provide a dynamic time depends upon the density of traffic. The disadvantage of green wave is it does mention the method which is used for communication between the emergency vehicle and the traffic signal controller. The green wave technology uses a rfid and a gps for automatic lane clearance for ambulance. The main aim of the system is to reduce the delay which required by the ambulance to reach the hospital. This will done by automatically clearing the lane in which ambulance is travelling before it reaches the traffic signal. When the ambulance is at particular distance the green light will glow and red light will off automatically of the particular lane. With the help of trans-receiver and GPS the communication between ambulance and traffic signal post takes place. It does not required human intervention at the traffic junction because it is fully automatic. The disadvantage of this system is it will needs all the information about the starting point, end point of the travel. The system will not work if starting point is unknown. Traffic is a critical issue of transportation system in most of all the cities of Countries. The traffic congestion is shown in figure 1. In the Bangalore city the growth of the population of vehicles increases exponentially due to which many intersections are overloaded, that reduces the speed of vehicles below acceptable speed. 


\section{PROPOSED SYSTEM}

The existing technologies are insufficient to handle the problems of emergency vehicle clearance. To solve such problems, Intelligent Traffic Control System is implemented. ZigBee transmitter module is placed in each emergency vehicle. ZigBee receiver module is placed at traffic junction. The switch will be switched ON when the vehicle is used for emergency purpose. This will send the signal through the ZigBee transmitter to the ZigBee receiver. As the signal sends to the traffic post the red light will turn into green light. After passing through the traffic signal Zig ee module will automatically gets off and green light will turn into red light.

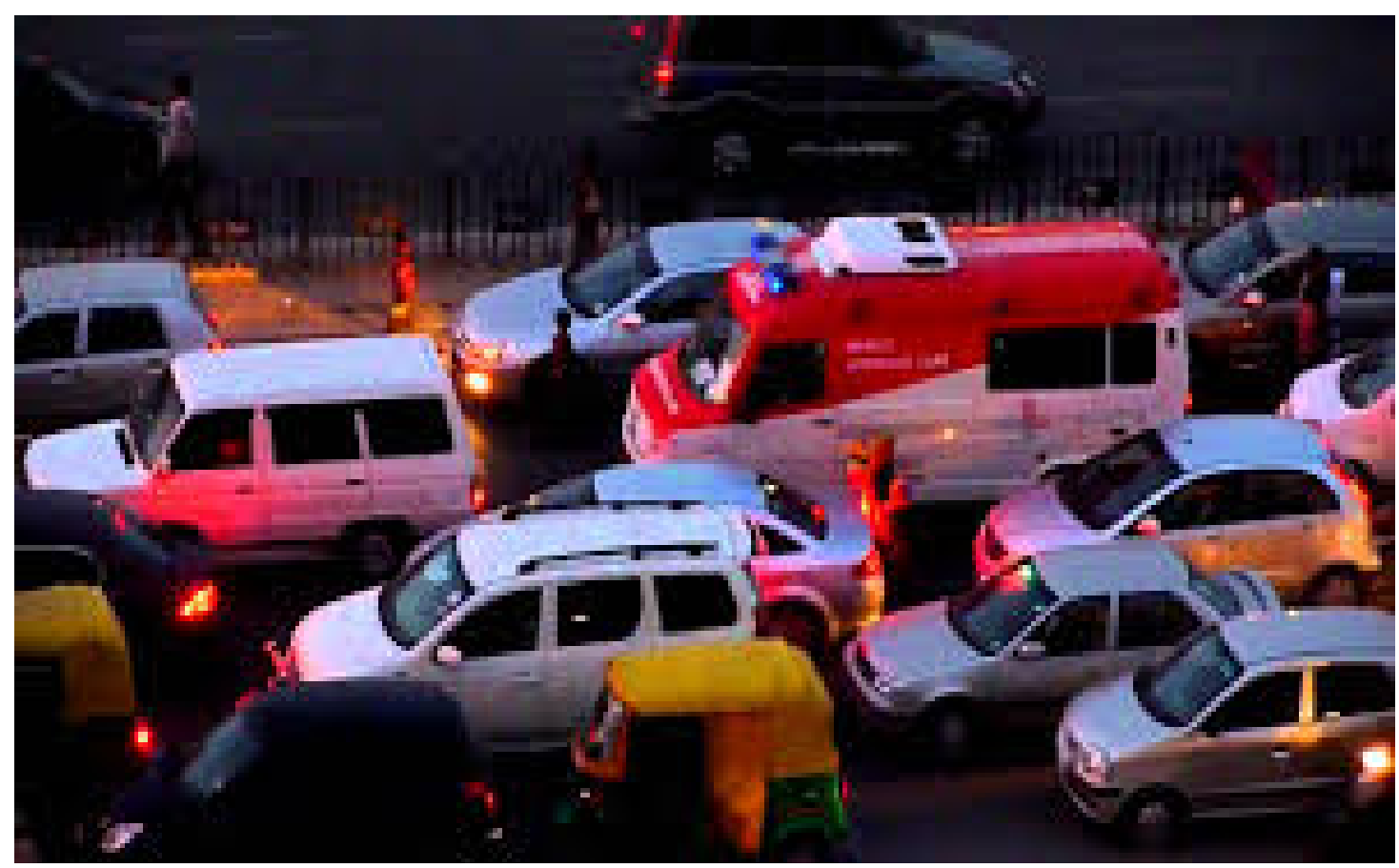

Figure 1: Drawback of current trafic control system

The CC2500 is a zigbee module and has transreceiver, which provides easy way to use communication at2.4 GHz. Every CC2500 is equipped with the microcontroller (PIC AT89C2805), which contains Unique Identification Number (UIN). The registration number of the vehicle is act as the UIN for that particular vehicle. The Unique Identification Number (UIN) is based on the serial communication without need of any extra hardware and extra coding. The role of trans-receiver is to provide communication in both directions. The microcontroller and CC2500 always communicate with the microcontroller via serial communication. Receiver (Rx) pin of CC2500 is connected with transmitter (Tx) (RC6) of microcontroller and Tx pin of CXC2500 is connected with Rx pin of microcontroller (RC7).Other two pins are used for activating trans-receiver. Figure shows the image of trans-receiver. Here, we uses CC2500 Zig-Bee module and it has transmission range of 10 meters. In this module, for experiment purpose, we have used passive ZIG-BEE tags and ZIG-BEE reader with frequency 2.4GHz. ZIG-BEE tag, when vehicle comes in the range of the receiver will transmit the unique ZIG$\mathrm{BEE}$ to the reader. The microcontroller connected to the ZIG-BEE reader will count the ZIG-BEE tags read-in 2 minute duration.

In transmitter section Power supply is given through $12 \mathrm{v}$ battery .The signal passes through 1000uF Capacitor for filtering signal. Then 7805 IC will convert $12 \mathrm{v}$ voltage to $5 \mathrm{v}$. The $470 \mathrm{uF}$ capacitor used for charging to give continuous flow of current in case of loading .The $5 \mathrm{v}$ voltage will be given as input to microcontroller initially the microcontroller requires to be reset, which will lead to 0 bit to all the ports. At port3, four switches are connected which will indicate that from which direction ambulance is coming and that direction will be send to the ZIG-BEE serially through MAX232.MAX232 used to convert 5v signal to 12v i.e. TTL to CMOS and viceversa. 


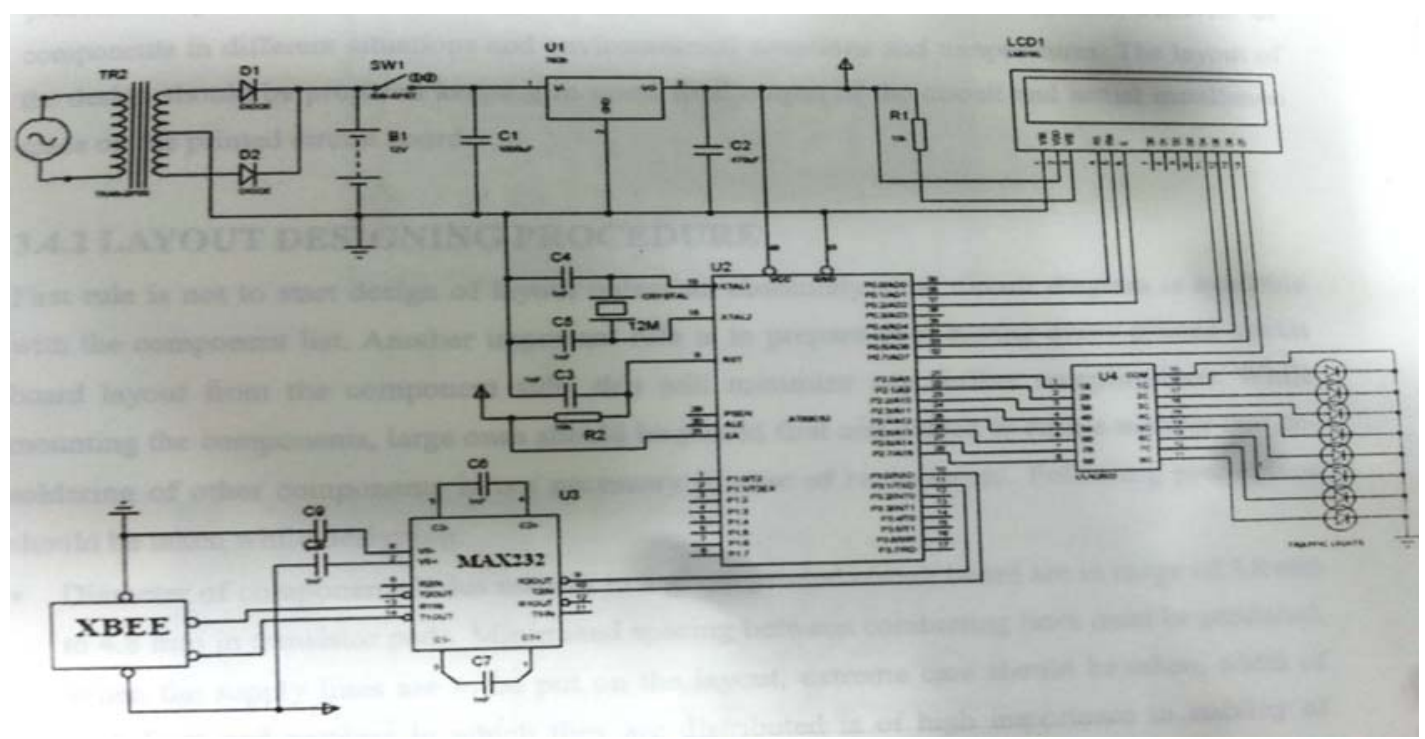

Figure 2: Trafic light controller with wireless transmitter

In the receiver section transformer will convert $230 \mathrm{v}$ to $12 \mathrm{v}$ AC. Rectifier is used to convert AC signal to DC signal. The working will be the same as transmitter in addition, At port 2 of PIC microcontroller LED's are connected for traffic signal. At port 0 LCD is connected which receives the data and display it, simultaneously PIC microcontroller monitors the signals coming from ZIG-BEE as well as manage timers to light-up directional signals.

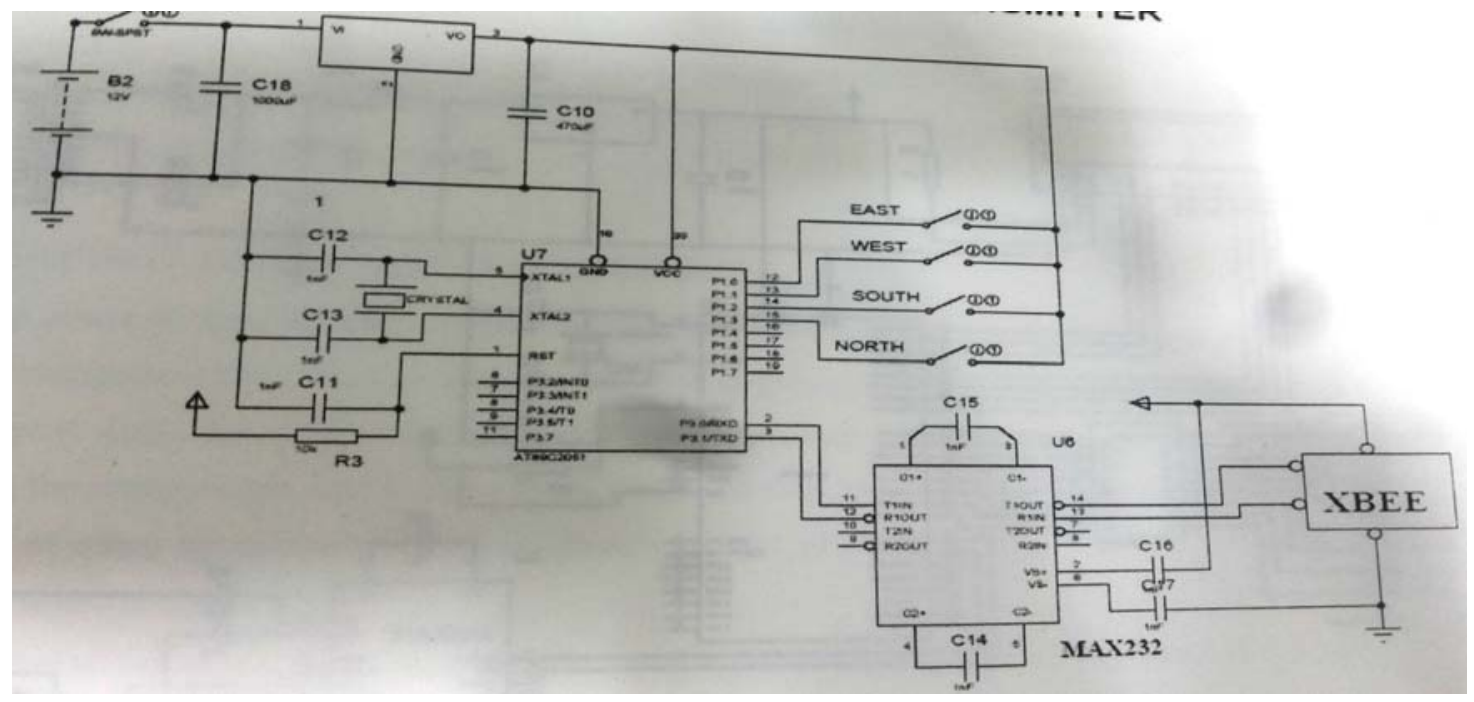

Figure 3: Ambulance controller with wireless transmitter

\section{CONCLUSION}

This type of Intelligent traffic control system is use to reduce the traffic in the way of ambulance. With the help of traffic synchronization the controlling of trimming is easily achievable. The designed system has acceptable results.

\section{REFERENCES}

[1] Rajeshwari Sundar, Santhoshs Hebbar, and Varaprasad Golla, "Implementing Intelligent Traffic Control System for, Ambulance Clearance”, IEEE Sensors Journal, vol. 15, no. 2, 2015.

[2] M. Abdoos, N. Mozayani, and A. L. C. Bazzan, "Traffic light control in non-stationary environments based on multi agent Qlearning,” in Proc. 14th Int. IEEE Conf. Intell. Transp. Syst., Oct. 2011, pp. 580-1585.

[3] K. Sridharamurthy, A. P. Govinda, J. D. Gopal, and G. Varaprasad, "Violation detection method for vehicular ad hoc networking" , Security Commun. Netw., to be published. Online available http://onlinelibrary.wiley.com/doi/10.1002/sec.427/abstract. 